\title{
Questões da Clínica Ginecológica que Motivam a Procura de Educação Médica Complementar: um Estudo Qualitativo
}

\author{
Clinical Issues in Gynecology that Motivate \\ the Search for Complementary Medical \\ Education: a Qualitative Study
}

Silvia Nogueira Cordeiro Paulo Cesar Giraldo Egberto Ribeiro Turato ${ }^{I I}$

\author{
PALAVRAS-CHAVE \\ - Psicologia Médica. \\ - Relação Médico-Paciente. \\ - Pesquisa Qualitativa. \\ - Ginecologia. \\ - Psicanálise. \\ - Psicologia.
}

Recebido em: 18/09/2009 Aprovado em: 05/11/2009

\begin{abstract}
RESUMO
Objetivo: Identificar questões emocionais subjetivas da consulta ginecológica que possam interferir na relação médico-paciente e motivar a necessidade de ampliar a formação pessoal como uma atividade complementar. Método: Foi realizado um estudo clínico-qualitativo de pesquisa, por meio da técnica de entrevista semidirigida de questões abertas. Estas foram submetidas à técnica de análise temática de conteúdo e elaboração de categorias de análise embasadas no referencial psicodinâmico. Foi constituída uma amostra intencional com oito médicos ginecologistas da Região Sudeste do Brasil. Resultados: A análise das entrevistas originou duas categorias temáticas sobre a relação médico-paciente: a consulta médica como "um encontro afetivo-profissional" e como "um momento de reflexão pessoal". Observou-se que a interação médico-paciente exerce importante papel na satisfação do exercício profissional, contribuindo para que o médico reflita sobre suas questões pessoais. Houve uma demanda por ensinamentos complementares que pudessem dar continência aos sentimentos internos mobilizados a partir da relação com a paciente. Há momentos de maior vulnerabilidade do médico, e o início da prática clínica é considerado um dos momentos marcantes de transição. Conclusão: Os entrevistados evidenciaram múltiplos aspectos que apontam uma forte mobilização de questões emocionais a partir do contato com as pacientes no ambiente da consulta médica. Limitações da educação médico-acadêmica dos ginecologistas entrevistados levaram à busca de complementação de sua formação pessoal, eventualmente incluindo suporte psicoterápico.
\end{abstract}

\begin{abstract}
Objective: The aim of this study was to identify subjective emotional issues in gynecological consultations that can interfere in the doctor-patient relationship and that raise the need for the gynecologist to seek complementary training. Method: A qualitative clinical research study was performed, using a semi-directed interview technique with open questions. The answers were submitted to thematic content analysis and elaboration of analytical categories based on the psychodynamic frame of reference. An intentional sample was used, including eight gynecologists from Southeast Brazil. Results: Analysis of the interviews revealed two thematic categories in the doctor-patient relationship: the medical appointment as "an affective-professional encounter" and as "a moment for personal reflection". According to the findings, doctor-patient interaction plays an important role in professional satisfaction, helping physicians reflect on their personal issues. There was a demand for complementary learning to contain the internal feelings mobilized by the relationship with the patient. There are moments of increased vulnerability for the gynecologist, and early clinical practice is considered one of the most striking moments of transition. Conclusion: The interviewees displayed various characteristics that suggest intense mobilization of emotional issues during contact with patients in the setting of the medical appointment. Limitations in the basic medical education of the gynecologists interviewed here motivated their search for complementary personal training, in some cases including psychotherapeutic counseling.
\end{abstract}

${ }^{I}$ Universidade Estadual de Londrina, Londrina, PR, Brasil; Universidade Estadual de Campinas, Campinas, SP, Brasil.

II Universidade Estadual de Campinas, Campinas, SP, Brasil. 


\section{INTRODUÇÃO}

A educação profissional para a prática da clínica ginecológica usualmente se limitou ao aprendizado sobre (dis)funções reprodutoras da mulher e elaboração dos diagnósticos médicos e respectivas condutas terapêuticas. Assim, tem havido pouco espaço para refletir sobre os aspectos subjetivos presentes na relação médico-paciente, que são elementos capazes de influenciar a consciência dos profissionais de saúde e de repercutir em suas intervenções, na melhoria dos serviços médicos e nas condições de vida tanto dos pacientes quanto dos médicos ${ }^{1}$.

Apesar dos crescentes recursos biotecnológicos, os conhecimentos básicos e as habilidades pessoais dos médicos continuam sendo o fundamento de sua atividade profissional. Porém, a prática da medicina contemporânea ainda apresenta barreiras que se revelam no encontro singular entre cada médico e sua paciente. Existe uma tendência atual de valorizar as sutilezas que se estabelecem no contato interpessoal e no vínculo deste binômio, que poderá ajudar ou dificultar o diagnóstico e o tratamento ${ }^{2}$

Durante o ensino acadêmico e principalmente ao exercerem a profissão, os médicos perceberam que a responsabilidade profissional constitui uma barreira importante de expressão no contato com a paciente. Muitas vezes, a teoria e a prática são insuficientes para atender às demandas subjetivas que surgem na interação que se estabelece numa consulta ginecológica. Para fazer o diagnóstico e instituir o tratamento adequado, não basta o conhecimento técnico, é necessário considerar que haverá um vínculo afetivo espontâneo entre ambos. Esse campo da subjetividade permite redescobrir uma linguagem que foi "esquecida" em virtude de uma educação predominantemente técnica ${ }^{3}$. Daí a importância das diretrizes curriculares nacionais, que apontam a necessidade de uma reflexão interna que propicie ao profissional o desenvolvimento de suas atitudes e habilidades para favorecer o vínculo. $\mathrm{O}$ desenvolvimento destas atitudes potencializa a empatia, a responsabilidade e a autonomia. Se o conhecimento científico é essencial para a construção de hipóteses, a prática clínica é essencial para exercitar a capacidade de interação com o meio e para construir o vínculo com a paciente ${ }^{4}$.

As experiências emocionais vividas a partir do contato com pacientes são essenciais a todas as áreas da medicina e à prática em saúde em geral, porém adquire singularidade no que diz respeito às características da personalidade de cada profissional e de cada paciente.

Neste sentido, poderíamos dizer que a prática gineco-obstétrica é uma especialidade singular, pois necessita lidar não só com os problemas orgânicos, mas também com tudo o que envolve a condição feminina, sexualidade e outras questões afetivas. Relembramos Balint ${ }^{5}$ (1984) ao dizer que na relação médico-paciente, um remédio fundamental é o próprio médico, que, como os demais medicamentos, precisa ser conhecido em sua posologia, efeitos colaterais e toxicidade. Deste modo, os fenômenos que ocorrem na consulta não deveriam ser focados apenas na paciente, mas também no médico. A Ginecologia é uma das especialidades em que o acompanhamento da paciente é feito por vários anos, favorecendo o vínculo estreito entre ela e o médico.

Pesquisas qualitativas realizadas com médicos ginecologistas que recentemente haviam concluído a residência médica demonstraram que, para eles, começar a atividade de consultório não é fácil, devido à insegurança. Estes profissionais ressaltaram a importância de terem o apoio de uma equipe multiprofissional durante o período de formação ${ }^{6}$.

Durante a formação especializada, entre elas as residências e os estágios, não existe, habitualmente, uma organização formal que oriente o ginecologista a lidar com situações subjetivas emocionais emergentes na consulta ginecológica. Considerando-se as particularidades inerentes a esta especialidade, supõese que, em diferentes níveis e com variadas intensidades, o ginecologista está sujeito às mobilizações internas resultantes do contato estabelecido com suas pacientes. Sabendo-se que a formação destes profissionais é provavelmente insuficiente para satisfazer as demandas a que são expostos, questiona-se se haveria na prática dos profissionais a necessidade de formação complementar que os subsidie para exercer a profissão ${ }^{7,8}$.

O objetivo deste trabalho foi analisar se ginecologistas identificam questões emocionais subjetivas na consulta ginecológica que possam interferir na relação médico-paciente e se sentem necessidade de ampliar sua formação pessoal não técnica como uma atividade complementar.

\section{SUJEITOS E MÉTODO}

Este estudo clínico-qualitativo analisou a percepção de oito ginecologistas (cinco homens e três mulheres) com mais de dez anos de prática profissional, com a finalidade de interpretar os sentidos e significados trazidos pelos sujeitos sobre os múltiplos fenômenos no campo das relações interpessoais da consulta ginecológica9.

Foi utilizada uma amostra intencional, ou seja, sujeitos com informações relevantes sobre o assunto a ser focaliza$\mathrm{do}^{10}$. Portanto, foram convidados a participar do estudo profissionais que, além de exercerem a prática clínica, atuam no ensino acadêmico ou trabalham com políticas de intervenção na Saúde Pública. Os sujeitos da amostra compuseram um grupo que foi fechado pelo critério de saturação das informações colhidas ${ }^{11}$. Os critérios de inclusão foram: (a) ser médico 
com residência médica já concluída em Ginecologia; (b) concordar em participar da entrevista ao tomar conhecimento de seu tema e do termo de consentimento livre e esclarecido; (c) aceitar que a entrevista fosse gravada e transcrita literalmente; (d) ter um segundo encontro com a pesquisadora se fosse necessário. $\mathrm{O}$ instrumento utilizado foi entrevista semidirigida com questões abertas, desenvolvidas a partir de um roteiro básico, porém sem rigidez, permitindo ao entrevistador fazer as necessárias adaptações a partir das informações obtidas do entrevistado ${ }^{12}$. Esta técnica permite que os entrevistados falem sobre os significados que atribuem ao fenômeno estudado em relação à sua vida. As entrevistas foram realizadas individualmente por apenas uma pesquisadora, em dias e horários escolhidos pelos entrevistados, e duraram cerca de 50 minutos. Os sujeitos foram identificados pelo número de ordem em que as entrevistas aconteceram. Estas foram realizadas nos respectivos locais de trabalho. Após estabelecimento do vínculo, a entrevista se iniciava com a pergunta "disparadora": "Conteme sobre a sua prática clínica e como na sua percepção se estabelece a relação médico-paciente?".

$\mathrm{Na}$ análise do material obtido nas entrevistas, foi utilizado o procedimento da análise temática, que consiste em desvelar os núcleos de sentido que presentes em uma cuja presença ou frequência tragam significado para o objetivo do analítico do estudo $^{13}$. Há uma ruptura entre o senso comum e o conhecimento acumulado através da atividade científica ${ }^{14}$.

A validação externa da análise das entrevistas foi discutida periodicamente com os pares do Laboratório de Pesquisa Clínico-Qualitativa do Departamento de Psiquiatria e Psicologia Médica da Universidade Estadual de Campinas, São Paulo, Brasil.

\section{RESULTADOS E DISCUSSÃO}

Após análise das entrevistas, juntamente com a leitura e a releitura flutuante, duas categorias temáticas foram construídas:

\section{A consulta médica: um encontro afetivo-profissional}

Os depoimentos demonstram que há preocupação dos ginecologistas com o manejo na consulta no intuito de estabelecer um vinculo afetivo-profissional. Alguns recortes das falas dos entrevistados exemplificam as proposições acima:

O vínculo depende do que a paciente vem preparada para ver de você. Às vezes ela já vem preparada para gostar de você. Outras vezes ela vem preparada para não gostar de você. Você tenta fazer com que ela goste, mas muitas vezes você não agrada. $\left(02-0^{7}\right)$

Eu identifico que existem expectativas muito diferentes na dependência de quem me procura [...]. na medida em que eu não faço parte da linha hegemônica, que meu discurso é um discurso contra-hegemônico [...] quer dizer, já tem uma identificação muito grande, pré-consulta. $\left(03-0^{\prime \prime}\right)$

Existem expectativas por parte destes profissionais sobre o tipo de vínculo que irão estabelecer com a paciente. Mesmo que o médico ocupe um lugar predeterminado social e culturalmente, que lhe propicia prestígio, ele detém determinado tipo de saber e, muitas vezes, é reconhecido como autoridade. Desse modo, vê-se que a subjetividade no campo de atuação ultrapassa a competência técnica ${ }^{15}$.

Fazendo-se uma leitura atenta destas falas, pode-se dizer que, em princípio, a consulta médica desperta nestes profissionais fantasias sobre o conjunto de fatores preexistentes à formação do vínculo, e estas fantasias podem contribuir ou não para estabelecer uma relação médico-paciente favorável à própria dupla.

$\mathrm{Na}$ atividade clínica extra-hospitalar e mesmo nas hospitalares, é a dinâmica do encontro com a paciente que irá formalizar o exercício profissional. O médico está submetido à autonomia da sua paciente, pois em última instância é ela quem o elege e decide sobre seu comprometimento com os procedimentos terapêuticos. Ainda que em determinados contextos, como, por exemplo, nos serviços públicos, a paciente, na maioria das vezes, não possa eleger diretamente seu médico, a adesão ao tratamento deixa implícito um sim ao vínculo, que possibilita ou não a continuidade do tratamento com "aquele médico".

Num estudo sobre a relação médico-paciente, Caprara e Rodrigues ${ }^{16}$ observaram que no começo da consulta quase todos os médicos estabeleceram uma relação empática com a paciente. Apesar disso, uma série de problemas surgiu de forma evidente, e foi observado que no decorrer da consulta a comunicação passou a ser unilateral.

Pode-se pensar que uma das dificuldades para o estabelecimento do vínculo surge quando o médico não dá a devida atenção aos problemas subjetivos da paciente, atendo-se única e exclusivamente aos aspectos técnicos da doença.

Atualmente, na sociedade ocidental, a visão tem sido mais centrada no individualismo e, como consequência, o hábito de escutar tem sido pouco enfatizado no cotidiano das interações entre os seres humanos ${ }^{17}$.

Por outro lado, nem sempre os profissionais percebem seus próprios elementos que facilitam ou bloqueiam o vínculo, como diz um dos entrevistados.

Se nós, ginecologistas, tivermos um ouvido competente, desenvolvermos uma escuta competente, afetiva, e 
aprendermos a não fazer juízo de valor, aprendermos a estar disponíveis para que estas mulheres possam se aproximar de nós, e fazer daquele espaço da consulta um espaço protegido, um espaço completo da sua relação médico-paciente. (05 -\$)

Segundo Entralgo ${ }^{18,19}(1978,1994)$, a relação médico-paciente é uma forma singular de amizade entre seres humanos, que deve abranger a benevolência, a benedicência, a beneficência e a benefidecência, que é a fusão - expansão de afeto - em direção ao outro para compartilhar com ele algo que lhe pertence intimamente, que se dá por confidência e vai permitir aos dois transformar a relação dual em dialética.

Para estabelecer relações construtivas com diálogo e uma verdadeira relação interpessoal, é necessário valorizar o "outro" em sua identidade, respeitando seus valores e compartilhando suas experiências ${ }^{20}$. Portanto, para surgir algo novo na relação médico-paciente, é necessário que os profissionais da saúde, em especial o médico, mudem de lugar, do saber a priori para um "não saber", no sentido de poder ouvir a paciente e construir um contato singular, pautado pelo desenvolvimento de habilidades cognitivas e afetivas que fortaleçam a escuta, o não julgamento moral. Esse depoimento traz à luz a necessidade de que o processo de ensino-aprendizagem tenha como eixo a questão afetiva, reforçando os esforços de mudança na formação do médico em nível de graduação e apontando para sua transformação em nível de pós-graduação em sentido lato.

\section{O contato com a paciente: um momento de reflexão pessoal}

$\mathrm{Na}$ vida tanto do médico quanto dos pacientes, há momentos naturais de maior vulnerabilidade, associados geralmente a períodos de transições existenciais. A finalização da educação acadêmica e o início da prática clínica podem ser considerados um destes momentos de transição.

Porém, mesmo depois da graduação, com certa experiência adquirida no manejo clínico, os médicos ainda são surpreendidos por mobilizações despertadas a partir desta relação, como exemplificado no próximo relato:

Eu sinto que o momento da consulta ginecológica é um momento que você reflete também suas coisas pessoais. Quando eu comecei minha vida profissional, eu não sabia nada do meu corpo, sabia muito pouco da minha sexualidade, e eu via que eu aprendia muito com as mulheres. $(04-q)$

Às vezes, quando eu termino o consultório, eu penso: Nossa, que dia! Eu estou carregada de ter ouvido o dia inteiro problemas das pacientes, não relacionados à saúde, mas problemas pessoais com o marido, com o filho.
É uma sucessão de problemas pessoais que as pacientes trouxeram, elas saíram ótimas, mas acabaram comigo. $(06-9)$

O contato sistemático por um longo período com a mesma paciente propicia maior proximidade na relação. Nem sempre o médico percebe que sua atuação é influenciada por seu estado emocional. As emoções que ele vivencia são suficientemente fortes para alterar de maneira substancial seus próprios padrões de conduta.

Por outro lado, as experiências adquiridas por meio da prática e nos atendimentos clínicos, muitas vezes, se encaixam em padrões que podem ser conjeturados, ainda que algumas situações clínicas sejam imprevisíveis. Nos depoimentos dos entrevistados, podemos observar que as consultas fazem emergir emoções e dificuldades, algumas das quais envolvem situações específicas que podem gerar o confronto com valores pessoais.

Eu procurei terapia pessoal logo no começo da carreira, porque uma pessoa me procurou querendo abortar, era uma aluna da faculdade. E eu falei assim: "Eu não sei o que fazer, não sei se eu te encaminho para outro médi$\mathrm{co}$, se eu fico furioso com você porque veio me procurar para interromper uma gravidez. Não sei se eu te ponho no meu colo para você chorar e para eu te acolher. Quer dizer... eu tenho mil dúvidas. Não posso te ajudar porque estou me sentindo pior que você. $\left(08-\sigma^{7}\right)$

Ainda que trate de um contexto específico, este relato exemplifica como o médico se sente tocado por situações reais trazidas pelas pacientes.

A medicina é uma profissão que lida constantemente com questões de impasse para o ser humano, como os limites entre vida e morte, e tudo o que se relaciona com o corpo e a sexualidade $^{21}$. Existem situações específicas da clínica ginecológica difíceis de manejar, pois fazem eco com questões ético-morais. Os conceitos ou preconceitos socioculturais, como, por exemplo, a prática do aborto e a diversidade das orientações psicossexuais, podem se confrontar com a prática médica. Se estiver atento a estes aspectos, o profissional sentirá necessidade de trabalhar suas questões pessoais, como ressalta o depoimento anterior.

Portanto, o processo de cuidar presente na relação médico-paciente também envolve componentes éticos e estéticos de bem viver individual-coletivo em determinado ambiente, tendo em vista a complexidade e a diversidade das múltiplas interconexões que se constroem na história de vida de ambos. Há um diálogo de escuta e troca de universos culturais e afetivos, que resulta em transformações tanto para o paciente quanto para o médico ${ }^{17}$. 
Por outro lado, é possível inferir que, quando o profissional não percebe que certas situações da prática clínica o transportam para núcleos conflitivos de suas experiências pessoais, sua intervenção junto à paciente pode receber influências projetivas destes aspectos internos. Um dos entrevistados reportou sobre este assunto:

\begin{abstract}
A formação médica precisa ser social e cultural, além de ética. Nós precisamos ter, minimamente, um discurso mais afinado, não pode ser uma coisa individual de cada ginecologista. Se um ginecologista tem uma educação moral, diferente, não pode julgar a mulher e tratá-la mal, ou então machucar durante o exame porque ela fez algo de que o médico discorda. (05 — \&)
\end{abstract}

Sabemos que a ação do médico como agente de saúde se amplia na medida em que ele evita confundir aspectos pessoais com as situações da prática clínica. As entrevistas identificaram a necessidade de incluir novos recursos teórico-práticos na formação médica a fim de trabalhar as demandas subjetivas emergentes na interação médico-paciente, motivando estes profissionais a buscar formação pessoal complementar:

[...] procurei estudos em saúde pública, em homeopatia e em sexualidade. Isso me ajudou muito na minha experiência de vida como mulher e como ginecologista. Eu realmente sinto uma tranquilidade porque fiz tais estudos e fui trabalhar minhas questões pessoais. (05 — +$)$ Foi uma procura mais marginal, não no esquema oficial. No esquema oficial, a gente não encontra praticamente nada, nem revistas médicas de ginecologia. Quando há alguma abordagem, são abordagens superficiais e fora da realidade concreta. Eu fiz uma procura minha mesmo. Eu acho que os processos terapêuticos que vivenciei também foram importantes. (04 -q)

Estudos atuais sobre a educação médica e relação médico-paciente apontam a necessidade de o estudante de Medicina ter professores com quem possa discutir questões pertinentes ao manejo da relação com a paciente, uma vez que ele percebe que é fortemente mobilizado por esse contato ${ }^{17,22-24}$.

A vulnerabilidade psicológica do médico é considerada um tema delicado, que se apresenta em função das pressões inerentes à profissão, no exercício diário com vida, doença e morte; pelo contato íntimo com o corpo do outro, com suas queixas, reivindicações, submissão e hostilidade; e pelas incertezas da medicina ${ }^{6}$.

Estudo realizado com médicos residentes em Ginecologia-Obstetrícia numa instituição pública no Brasil mostrou que, embora os entrevistados tenham considerado esta especialidade mais ligada à vida do que à doença, e consequentemente, à morte, quando essa especialidade envolve casos graves, principalmente em Obstetrícia, provoca impacto e angústia muito grandes ${ }^{25}$.

Hoje, as escolas médicas brasileiras estão encorajadas a rever seus projetos pedagógicos. Por este motivo, acreditamos que este trabalho é oportuno para reforçar a importância de construir parâmetros mais bem delineados dos conteúdos e habilidades que serão desenvolvidos na formação médica, para que seja possível a construção da subjetividade desses profissionais, principalmente quando optam por uma especialidade, como a Ginecologia, cuja prática permeia constantemente questões pertinentes à identidade e à sexualidade, pilares da personalidade.

Conclui-se, portanto, que os entrevistados evidenciaram múltiplos aspectos que apontam uma forte mobilização de questões emocionais do ginecologista a partir do contato com as pacientes no ambiente da consulta médica. Identificaram-se limitações da educação médico-acadêmica dos ginecologistas, que os levaram à busca de formação pessoal complementar, eventualmente incluindo suporte psicoterápico.

Diante dessas conclusões, os autores destacam a importância de implementar, nos programas de residência na área da Ginecologia, uma abordagem psicopedagógica que possa dar subsídios aos dilemas emocionais que envolvem a atuação do médico na prática clínica nessa especialidade. Neste sentido, criar um ambiente nas discussões de casos clínicos em que as questões psicodinâmicas da relação do médicopaciente sejam abordadas adequadamente seria um fator importante, assim como estimular o ginecologista a procurar atendimento psicoterápico quando for diagnosticada a necessidade.

\section{AGRADECIMENTOS}

Os autores reconhecem e agradecem a cooperação dos sujeitos entrevistados. Reconhecem também as contribuições feitas ao longo da revisão pelos pares do Laboratório de Pesquisa Clínico-Qualitativa, da Faculdade de Ciências Médicas, Universidade Estadual de Campinas.

\section{REFERÊNCIAS}

1. Caprara A, Franco ALS. A relação médico-paciente para uma humanização da prática médica. Cad Saúde Pública. 1999;15(3):647-54.

2. Bastos LAM. Corpo e subjetividade: um diálogo da psicanálise com a medicina. Rev Bras Psicanál. 2003;37(1):145-58.

3. Berg M. Racionaliting Medical Work: Decision-support Tecniques and Medical Practices. Cambridge, Massachussetts: MIT Press; 1997. 
4. Schraiber LB. O médico e seu trabalho: limites da liberdade. São Paulo: Hucitec; 1993.

5. Balint M. O médico, seu paciente e a doença. Rio de Janeiro: Ateneu; 1984.

6. Gilbert ACB, Cardoso MHCA, Wuillaume SM. Médicos Residentes e suas relações com/e no mundo da saúde e da doença: um estudo de caso institucional com residentes em Obstetrícia/Ginecologia. Interface Comum Saúde Educ. 2006;10(19):103-16.

7. Ramalho MAN, Nogueira-Martins MCF. Vivências de profissionais de saúde da área de oncologia pediátrica. Psicol Estud. 2007;12(1):123-32.

8. Lima VV. Competência: distintas abordagens e implicações na formação de profissionais de saúde Interface Comum Saúde Educ. 2005;9(17):369-79.

9. Turato ER. Tratado da Metodologia da Pesquisa ClínicoQualitativa: Construção Teórico-pistemológica, Discussão Comparada e Aplicação nas Áreas da Saúde e Humanas. $3^{\text {á }}$ ed. Petrópolis: Vozes; 2008.

10. Patton MQ. Qualitative Research \& Evaluation Methods. $3^{\mathrm{a}}$ ed. Thousand Oaks: Sage Publications; 2001.

11. Fontanella BJB, Rica J, Turato ER. Abordagem por saturação em pesquisas qualitativas em saúde: contribuições teóricas. Cad Saúde Pública. 2008;24(1):17-24.

12. Fontanella BJB, Campos CJG, Turato ER. Coleta de dados na pesquisa clinico - qualitativa: uso de entrevistas não dirigidas de questões abertas por profissionais da saúde. Rev Lat Am Enfermagem. 2006;14(5):812-20.

13. Minayo MCS. O desafio do conhecimento: pesquisa qualitativa em saúde. São Paulo: Hucitec; 1999.

14. Turato ER, Machado AC, Silva DF, Carvalho GM, Verderosi NR, Souza TF. Research publications in the field of health: omission of hypotheses and presentation of commonsense conclusions. Sao Paulo Med J [periodic na Internet]. 2006 [acesso em 24 mar. 2008];124(4):228-33. Disponível em: http://www.scielo.br/pdf/spmj/v124n4/32074.pdf

15. Jeamment $P$, Reynaud M, Consoli SM. Manual Psicología Médica. 2ª ed Barcelona: Masson; 1999.

16. Caprara A, Rodrigues J. A relação assimétrica médicopaciente: repensando o vínculo terapêutico. Ciênc Saúde Coletiva. 2004;9(1):139-46.

17. Grosseman S, Patricio ZM. A relação medico-paciente e o cuidado humano: subsídios para promoção da educação médica. Rev Bras Educ Med. 2004;28(2):99-105.

18. Entralgo P. Historia de la medicina. Barcelona: Salvat; 1978.

19. Entralgo P. Técnica, ética y Amistad médica. In: Viveros MG, orgs. Medicina y sociedad. México: Fondo de Cultura Económica; 1994. p.27-36.
20. Guareschi P. Alteridade e relação: uma perspective crítica. In: Arruda A. Representando a alteridade. Petrópolis: Vozes; 1998. p. 149-61.

21. Nogueira-Martins LA. Saúde Mental dos Profissionais de Saúde. Rev Bras Med Trab. 2003;1(1):56-68.

22. Stark P. Teaching and learning in the clinical setting: a qualitative study of the perception of student and teachers. Med Educ. 2003;37(11):975-82.

23. Nogueira-Martins MCF, Nogueira Martins LA, Turato ER. Medical student's perception of their learning about the doctor-patient relationship: a qualitative study. Med Educ. 2006;40:322-8.

24. Sarton TCF, Fiorini VML. Programa de Orientação de Estudantes do Curso Médico Famema: Regatando, Reconstruindo e Buscando Bases para o Fortalecimento. Rev Bras Educ Med. 2004;28(2):173-7.

25. Nogueira-Martins LA, Jorge MR. Natureza e magnitude do estresse na Residência Médica. Rev Assoc Med Bras. 1998;44(1):28-34.

Apoio: Fonte de financiamento: Bolsa da Coordenação de Aperfeiçoamento de Pessoal de Nível Superior (Capes). Ministério da Educação do Brasil, 2004-2007.

\section{CONTRIBUIÇÃO DOS AUTORES}

Os três autores participaram da elaboração, análise e interpretação dos resultados encontrados, assim como da redação deste artigo. Sílvia Nogueira Cordeiro, pesquisadora responsável pelo projeto, realizou as entrevistas; Paulo César Giraldo contatou os ginecologistas para serem entrevistados e foi coorientador do trabalho e Egberto Ribeiro Turato foi orientador da primeira autora e planejou os recursos metodológicos

\section{CONFLITO DE INTERESSES}

Declarou não haver.

\section{ENDEREÇO PARA CORRESPONDÊNCIA}

Sílvia Nogueira Cordeiro

Rua Camargo Pimentel, 377

Jardim Guanabara - Campinas

CEP. 13073-340 - SP

E-mail: silvianc2000@hotmail.com 